\title{
INTERVALO HÍDRICO ÓTIMO COMO INDICADOR DE MELHORIA DA QUALIDADE ESTRUTURAL DE LATOSSOLO DEGRADADO(1)
}

\author{
Vico Mendes Pereira Lima ${ }^{(2)}$, Geraldo César de Oliveira ${ }^{(3)}$, Milson \\ Evaldo Serafim $^{(4)}$, Nilton Curi(5) \& Antônio Ricardo Evangelista ${ }^{(6)}$
}

\begin{abstract}
RESUMO
As pastagens de gramíneas, quando bem formadas e manejadas, representam um componente ambiental importante em razão do papel que exercem na cobertura dos solos, na formação e estabilização dos agregados e na redução do adensamento ou da compactação. Os objetivos deste estudo foram quantificar o intervalo hídrico ótimo (IHO), para um Latossolo Vermelho-Amarelo degradado fisicamente e cultivado com Coastcross (Cynodon spp.), e utilizar o IHO como indicador de alteração da estrutura desse solo. Amostras indeformadas foram coletadas em anéis volumétricos de $0,025 \mathrm{~m}$ de altura e $0,065 \mathrm{~m}$ de diâmetro, nas profundidades de 0-0,05 e 0,20-0,25 m e no horizonte $B w(0,80-0,85 \mathrm{~m})$, na ausência de tráfego de máquinas agrícolas, durante a estação chuvosa de 2008/2009, nos meses de novembro de 2008, março de 2009 e maio de 2009. Essas amostras foram utilizadas para determinar a curva de retenção de água, a curva de resistência à penetração, a densidade, o IHO e a densidade crítica do solo (Dsc). O IHO revelou-se um adequado indicador da alteração da estrutura de Latossolo. O Coastcross apresenta potencial para melhoria da estrutura, sendo sugerida sua utilização como alternativa ao uso de implementos agrícolas nesse contexto. A Dsc de $1,24 \mathrm{Mg} \mathrm{m}^{-3}$ é limitante ao adequado crescimento e desenvolvimento da forrageira no solo em estudo.
\end{abstract}

Termos de indexação: qualidade estrutural do solo, disponibilidade hídrica, resistência do solo, qualidade física do solo.

(1) Parte da Tese do primeiro autor apresentada ao Programa de Pós-Graduação em Ciência do Solo da Universidade Federal de Lavras - UFLA. Recebido para publicação em 17 de março de 2011 e aprovado em 19 de outubro de 2011.

(2) Professor do Instituto Federal de Educação, Ciência e Tecnologia do Norte de Minas - IFNMG. Campus Almenara, CEP $39900-000$ Almenara (MG). Email: vico.lima@ifnmg.edu.br

(3) Professor do Departamento de Ciência do Solo, Universidade Federal de Lavras - UFLA. Caixa Postal 3037, CEP 37200-000 Lavras (MG). E-mail: geraldooliveira@dcs.ufla.br

(4) Professor do Instituto Federal de Educação, Ciência e Tecnologia de Mato Grosso, Campus Cáceres, CEP 78106-960 Cáceres (MT). E-mail: milsonserafim@gmail.com.

(5) Professor do Departamento de Ciência do Solo, UFLA. E-mail: niltcuri@dcs.ufla.br

(6) Professor do Departamento de Zootecnia, UFLA. E-mail: aricardo@dzo.ufla.br 


\title{
SUMMARY: LEAST LIMITING WATER RANGE AS INDICATOR OF STRUC- TURAL QUALITY IMPROVEMENT OF A DEGRADED LATOSOL (OXISOL)
}

\begin{abstract}
Grass pastures, if well-established and managed, play an important environmental role as soil cover, for aggregate formation and stabilization and in the reduction of dense or compacted layers. The objectives of this study were to quantify the least limiting water range (LLWR) of a physically degraded Red-Yellow Latosol (Oxisol) on which bermudagrass (Cynodon spp., cultivar coastcross) was planted, and to use the LLWR as an indicator of changes in the soil structural quality. Undisturbed samples were collected in volumetric rings (height $0.025 \mathrm{~m}$, diameter $0.065 \mathrm{~m}$ ) from the layers $0-0.05,0.20-0.25$ and $0.80-0.85 \mathrm{~m}$ (Bo horizon), in areas without machine traffic, during the rainy season of 2008/2009 in november 2008, march 2009 and may 2009. Based on these samples, the water retention curve, penetration resistance curve, bulk density, LLWR, and soil critical density were determined. The LLWR proved to be an adequate indicator of changes in the Latosol structure. The grass cultivar coastcross is potentially suitable for improving the soil structural quality, and is suggested for this purpose, instead of agricultural implements. The soil critical density of $1.24 \mathrm{Mg} \mathrm{m}^{-3}$ restricts the adequate development of the studied grass root system.
\end{abstract}

Index terms: soil structural quality, water availability, soil resistance, soil physical quality.

\section{INTRODUÇÃO}

A boa qualidade das forrageiras conservadas contribui para um manejo animal equilibrado, com suprimento de nutrientes e com maior estabilidade durante todo o ano. Entre os processos de conservação de forrageiras, destaca-se a fenação, na qual é empregada a mecanização intensiva em todas as etapas de produção (Giarola et al., 2007). Apesar dessa vantagem, existem limitações relacionadas à compactação superficial do solo, originada pelo tráfego de máquinas e equipamentos agrícolas. A compactação do solo modifica a quantidade, a continuidade e o tamanho dos poros (Richard et al., 2001; Klein \& Libardi, 2002; Oliveira et al., 2004), com reflexos na qualidade física do solo para crescimento e produtividade das plantas (Tormena et al., 2007).

A melhoria da estrutura do solo promovida pelas forrageiras (Severiano et al., 2010) constitui uma alternativa para o controle da compactação superficial e da qualidade física e estrutural do solo. $\mathrm{O}$ uso de plantas, que atuam nesse sentido, compõe uma importante estratégia de manejo em sistemas intensivos de produção (Jimenez et al., 2008), sendo a alta densidade e a periódica renovação das raízes de gramíneas alguns dos fundamentos para a qualidade e sustentabilidade da produção (Silva \& Mielniczuk, 1997).

Do ponto de vista físico, o uso de plantas que contribuem para a melhoria da estrutura do solo, como técnica de manejo, propicia a formação de bioporos com ampla variação de tamanho. Esses bioporos funcionam como rotas alternativas para o crescimento das raízes (Williams \& Weil, 2004) e aumentam o movimento de água e a difusão de gases no solo (Müller et al., 2001). Essas plantas apresentam potencial de redução do impedimento mecânico, além de contribuírem para a melhoria do estado de agregação do solo (Camargo \& Alleoni, 1997).

Condições físicas restritivas ao bom crescimento e desenvolvimento das plantas reduzem a capacidade produtiva dos solos e a sustentabilidade ambiental. As complexas e dinâmicas interações entre resistência do solo à penetração $(\mathrm{RP})$, aeração e disponibilidade de água alteram diretamente o crescimento e desenvolvimento das plantas. Com o intuito de integrar as principais propriedades físicas do solo determinantes do crescimento e desenvolvimento das plantas, Silva et al. (1994) propuseram o intervalo hídrico ótimo (IHO), com base nos pressupostos de Letey (1985), como um índice para avaliar a qualidade física do solo. A diminuição da amplitude do IHO indica o aumento da exposição das culturas ao estresse físico do solo e a magnitude na qual a condição estrutural restringe o crescimento e desenvolvimento das plantas (Silva et al., 1994). Em solos com reduzido IHO, as variações espaciais e temporais do conteúdo de água do solo podem predispor as culturas a estresses físicos, seja pela aeração deficiente em condições de solo muito úmido, seja pelo excessivo aumento da RP por ocasião do secamento do solo (Bengough et al., 2006).

Assim, o IHO representa ganhos em termos de biofísica do solo, sendo um indicador que reflete adequadamente a qualidade física do solo para as plantas (Tormena et al., 2007). Várias pesquisas 
realizadas em diferentes solos, culturas e sistemas de manejo comprovam a pertinência da aplicação do IHO na avaliação da qualidade física do solo (Kaiser et al., 2009; Lima et al., 2009; Medeiros et al., 2011).

No IHO, a faixa ótima de conteúdo de água do solo para o crescimento das plantas depende da densidade do solo (Ds) (Leão et al., 2005). Para cada valor de Ds, um valor de IHO é calculado e definido, a partir dos limites superior e inferior de água no solo. Geralmente, o aumento de Ds resulta em redução do IHO, em direção a valores de Ds em que o IHO se iguala a zero. A Ds em que o IHO = 0 é denominada de densidade crítica do solo (Dsc) (Silva et al., 1994; Imhoff et al., 2001). Quando Ds > Dsc, há indicações de acentuada degradação estrutural do solo, restringindo o crescimento das plantas. Os valores de Dsc variam em função do nível de compactação e da textura (Wu et al., 2003; Reichert et al., 2009), além da estrutura do solo. Potencialmente, sistemas de manejo que propiciam maior frequência de Ds < Dsc oferecem menores restrições físicas às plantas.

Estudos sobre o potencial de as raízes de gramíneas alterarem a qualidade física e estrutural do solo não são frequentes na literatura. No estádio atual de evolução dos sistemas agrícolas, em que se busca aprimorar a sua qualidade, é necessário conhecer as alterações impostas ao ambiente físico do solo pelo crescimento e renovação do sistema radicular. Assim, este estudo foi realizado com os objetivos de quantificar o IHO em diferentes profundidades e durante o período chuvoso do ano, para um Latossolo Vermelho-Amarelo degradado fisicamente e cultivado com Coastcross (Cynodon spp.), e utilizar o IHO como indicador de alteração da estrutura desse solo.

\section{MATERIAL E MÉTODOS}

A área experimental está localizada no Sítio Cata-Vento, município de Itutinga, Minas Gerais, na região sob influência do reservatório da hidrelétrica de Itutinga/Camargos (MG). A área do reservatório abrange os municípios de São João Del Rei, Itutinga, Nazareno, Carrancas, Madre de Deus de Minas, São Vicente de Minas, Andrelândia e Minduri, perfazendo um total de $2.084,4 \mathrm{~km}^{2}$. O clima local é classificado, segundo Köppen, como Cwa de verão brando e chuvoso, com temperaturas moderadas, e média anual de $21^{\circ} \mathrm{C}$. A precipitação pluvial média anual é de $1.500 \mathrm{~mm}$, e a média anual de umidade relativa do ar, de $70 \%$.

O estudo foi desenvolvido em Latossolo VermelhoAmarelo (LVA) textura muito argilosa, degradado fisicamente. O solo foi descrito e classificado em novembro de 2008 (Nov/08) segundo o Sistema Brasileiro de Classificação dos Solos (Embrapa, 2006), utilizando o Manual de Descrição e Coleta de Solo no Campo (Santos et al., 2005). Esse solo é bastante representativo da zona fisiográfica Campos das Vertentes e apresenta alto potencial para a utilização agrícola (Chagas et al., 1997). A análise granulométrica da profundidade de 0-0,05 m apresentou valores médios de 630, 240 e $130 \mathrm{~g} \mathrm{~kg}^{-1}$; da profundidade de 0,20-0,25 m, 670, 210 e $120 \mathrm{~g} \mathrm{~kg}^{-1}$; e da profundidade de $0,80-0,85 \mathrm{~m}$, 710,190 e $100 \mathrm{~g} \mathrm{~kg}^{-1}$ de argila, silte e areia, respectivamente.

O experimento foi implantado em parte da área de 9 ha cultivada com Coastcross (Cynodon spp.), visando à produção de feno. Na média, ocorrem 12 operações de tráfego mecanizado (corte, secagem, enfardamento e transporte) durante as etapas produtivas; essas operações são multiplicadas por três, quando se consideram os sucessivos cortes durante a estação chuvosa do ano. A área está sob esse manejo há aproximadamente oito anos. O experimento foi conduzido na ausência de tráfego de máquinas agrícolas, durante três ciclos produtivos do ano de 2008/09. As correções das deficiências nutricionais, nessa área, foram realizadas com a aplicação de $121 \mathrm{~kg} \mathrm{ha}^{-1}$ de N, $42 \mathrm{~kg} \mathrm{ha}^{-1}$ de P, e $117 \mathrm{~kg} \mathrm{ha}^{-1}$ de K.

A coleta de amostras indeformadas $(0,065 \mathrm{~m}$ de diâmetro por $0,025 \mathrm{~m}$ de altura) do solo foi realizada nos meses de novembro de 2008, março de 2009 e maio de 2009, nas profundidades de 0-0,05 e 0,20$0,25 \mathrm{~m}$ (15 amostras $\mathrm{x} 2$ profundidades $\mathrm{x} 3$ períodos $=90$ amostras) e na profundidade de $0,80-0,85 \mathrm{~m}$, correspondente ao horizonte $\mathrm{Bw}$ ( 15 amostras x 1 profundidade $\mathrm{x} 1$ período $=15$ amostras), realizada no mês de novembro de 2008, totalizando 105 amostras. Essas amostras foram envoltas em plástico filme, parafinadas e acondicionadas em sacos plásticos, sob temperatura de aproximadamente $22{ }^{\circ} \mathrm{C}$, até serem processadas no laboratório.

As amostras foram devidamente preparadas e saturadas por meio da elevação gradual de uma lâmina de água. Para determinação da curva de retenção de água (CRA), as amostras foram divididas em cinco grupos de 21 amostras, sendo três repetições em cada potencial utilizado. Foram usados os seguintes potenciais: 0, obtido a partir do volume total de poros; -0,006 $\mathrm{MPa}$, na unidade de sucção; $-0,033,-0,100$ e - $1,5 \mathrm{MPa}$, nas membranas extratoras de Richards (Embrapa, 1997); e o valor inferior a -1,5 até o limite de -60 MPa, no psicrômetro de termopar (WP4-T). Após atingirem o equilíbrio hídrico em cada potencial, as amostras foram pesadas, sendo determinada a RP, utilizando-se o penetrógrafo eletrônico de bancada, conforme Tormena et al. (1998). Após determinação da RP, as amostras foram secas em estufa a $105^{\circ} \mathrm{C}$, por $24 \mathrm{~h}$, para determinação da Ds e do conteúdo de água do solo 
$(\theta)$. A macroporosidade foi calculada pela diferença entre VTP $=(1-\mathrm{Ds} / \mathrm{Dp})$, e a microporosidade (água retida na tensão de $-0,006 \mathrm{MPa}$ ), segundo método descrito por Embrapa (1997).

$\mathrm{Na}$ determinação do IHO, foram consideradas a CRA e a curva de resistência do solo à penetração (CRS). A CRA foi obtida ajustando-se o $\theta$ em função do potencial matricial da água no solo $(\Psi)$, utilizando a equação 1 e o procedimento de van Genuchten (1980):

$$
\theta=\left(\theta_{\text {sat }}-\theta_{\text {res }}\right)\left[1+(\alpha \Psi)^{\mathrm{n}}\right]^{-\mathrm{m}}+\theta_{\text {res }}
$$

sendo $\Psi$ o potencial matricial da água no solo $(\mathrm{kPa})$; $\theta$, o conteúdo de água $\left(\mathrm{m}^{3} \mathrm{~m}^{-3}\right) ; \theta_{\text {sat }}$, o conteúdo de água na saturação $\left(\mathrm{m}^{3} \mathrm{~m}^{-3}\right)$; $\theta_{\text {res }}$, o conteúdo de água no ponto de murcha permanente $\left(\mathrm{m}^{3} \mathrm{~m}^{-3}\right)$; e $\mathrm{m}, \mathrm{n}$ e $\alpha$, os parâmetros de ajuste do modelo.

A CRS foi ajustada a modelos não lineares, em função do $\theta$, utilizando-se a equação 2 e o software Sigma Plot 10.0 (2006):

$$
\mathrm{RP}=\mathrm{a}+\mathrm{b} e^{(\mathrm{cDs})}
$$

sendo $\mathrm{RP}$ a resistência à penetração $(\mathrm{kPa}) ; \mathrm{a}, \mathrm{b}$ e c, os parâmetros de ajuste da equação; e $\theta$, o conteúdo volumétrico de água $\left(\mathrm{m}^{3} \mathrm{~m}^{-3}\right)$.

O IHO foi determinado adotando-se os procedimentos descritos em Silva et al. (1994) e Tormena et al. (1998). Os valores críticos de conteúdo de água do solo associados com o potencial mátrico, a resistência do solo à penetração e a porosidade de aeração foram, respectivamente: a umidade na capacidade de campo $\left(\theta_{\mathrm{CC}}\right)$ ou conteúdo de água estimado no potencial de -0,006 $\mathrm{MPa}$; a umidade no ponto de murcha permanente $\left(\theta_{\mathrm{PMP}}\right)$ ou conteúdo de água no potencial de -1,5 MPa; e o conteúdo de água do solo em que a porosidade de aeração $\left(\theta_{\mathrm{PA}}\right)$ é de $0,10 \mathrm{~m}^{3} \mathrm{~m}^{-3}$. Para a $R P$ foi adotado o valor crítico de $2,5 \mathrm{MPa}$, com base nos resultados de Camargo \& Alleoni (1997). Os valores de $\theta_{\mathrm{CC}}$ e $\theta_{\mathrm{PMP}}$ foram obtidos nos potenciais de - 0,006 e -1,5 MPa, utilizando a CRA; e os valores do teor de água em que a $R P\left(\theta_{\mathrm{RP}}\right)$ atinge o valor crítico de $2,5 \mathrm{MPa}$ foram obtidos por meio da CRS. O valor médio de densidade de partículas foi determinado nas amostras, conforme Embrapa (1997): Dp = $2,50 \mathrm{Mg} \mathrm{m}^{-3}$, para a profundidade de 0-0,05 e $0,20-0,25 \mathrm{~m}$; e $\mathrm{Dp}=2,66 \mathrm{Mg} \mathrm{m}^{-3}$, para o horizonte $\mathrm{Bw}(0,80-0,85 \mathrm{~m})$.

$\mathrm{Na}$ determinação dos limites superiores do IHO, considerou-se o $\theta_{\mathrm{CC}}$, ou aquele em que a $\theta_{\mathrm{PA}}$ é considerada adequada ao crescimento e desenvolvimento da cultura. Como limites inferiores foram considerados o $\theta_{\mathrm{PMP}}$ ou aquele correspondente à $\theta_{\mathrm{RP}}$ limitante ao crescimento e desenvolvimento das plantas, segundo critérios propostos por Silva et al. (1994).
Posteriormente, foram coletadas amostras indeformadas nos meses de agosto/09 e fevereiro/10 para determinação da Ds e macroporosidade segundo método descrito por Embrapa (1997).

A distribuição dos dados de Ds e macroporosidade no modelo de distribuição normal foi avaliada pelo teste de Shapiro-Wilk, utilizando o programa estatístico Sisvar, conforme Ferreira (2005). A análise de variância dos dados de Ds e macroporosidade foi realizada utilizando-se o modelo de delineamento inteiramente casualizado, com médias comparadas pelo teste de Scott-Knott $(\mathrm{p}<0,05)$. Os ajustes das equações das CRA e CRS foram feitos pelo método de regressão não linear, usando o software Sigma Plot 10.0 (2006). A comparação entre os limites superiores e inferiores do IHO foi realizada por meio das análises do erro-padrão da média.

\section{RESULTADOS E DISCUSSÃO}

$\mathrm{Na}$ descrição morfológica do solo realizada durante o mês de novembro de 2008 , verificou-se que a cobertura vegetal era descontínua, com reboleiras de plantas com mau crescimento e amareladas, além de presença de encrostamento na superfície do solo. O histórico de pressões aplicadas ao solo durante oito anos de manejo impôs alterações estruturais nos primeiros $0,42 \mathrm{~m}$, deixando a estrutura pouco porosa, diferentemente da estrutura do horizonte $\mathrm{Bw}$, que se manteve inalterada, tendo aspecto maciço poroso. Esses aspectos corroboram o trabalho de Giarola et al. (2007).

As plantas cresceram satisfatoriamente na ausência de tráfego de máquinas e implementos agrícolas, em condições adequadas de suprimento de água e nutrientes no solo, provenientes do manejo controlado e das adubações realizadas no período de chuva. Na avaliação temporal da Ds e da macroporosidade ao longo desse período, observaram-se valores menores de Ds e maiores de macroporosidade, estatisticamente diferenciados, para esses atributos no mês de maio de 2009 , em comparação a novembro de 2008 e março de 2009, independentemente da profundidade, o que indica a ocorrência de alívio na estrutura do solo (Quadro 1).

Os valores de Ds e de macroporosidade no mês de maio de 2009, nas duas primeiras profundidades, aproximaram-se da Ds e da macroporosidade do horizonte $\mathrm{Bw}$, que apresenta estrutura preservada. Isso pode ser atribuído ao potencial de melhoria da estrutura do solo por capins do gênero Cynodon (Magalhães et al., 2009). Dessa forma, acredita-se que o período para essa melhoria está associado à alta densidade do sistema radicular dessas gramíneas, ao tempo necessário para a morte e 
Quadro 1. Densidade (Ds) e macroporosidade do solo nas profundidades de 0-0,05 e 0,20-0,25 m em comparação ao horizonte Bw do Latossolo Vermelho-Amarelo sob Coastcross, no período chuvoso de 2008/2009

\begin{tabular}{cccc}
\hline Profundidade & Mês & Ds $^{(1)}$ & Macroporosidade $^{(2)}$ \\
\hline & & Mg m$^{-3}$ & $\mathrm{~m}^{3} \mathrm{~m}^{-3}$ \\
& Nov/08 & $1,22 \mathrm{~d}$ & $0,06 \mathrm{c}$ \\
$0-0,05 \mathrm{~m}$ & Mar/09 & $1,17 \mathrm{c}$ & $0,07 \mathrm{c}$ \\
& Maio/09 & $1,06 \mathrm{~b}$ & $0,16 \mathrm{~b}$ \\
$\mathrm{Bw}$ & & $0,97 \mathrm{a}$ & 16,47 \\
$\mathrm{CV}(\%)$ & & 4,32 & $0,12 \mathrm{c}$ \\
$0,20-0,25 \mathrm{~m}$ & Mov/08 & $1,16 \mathrm{c}$ & $0,12 \mathrm{c}$ \\
$\mathrm{Bw}$ & Maio/09 & $1,15 \mathrm{c}$ & $0,16 \mathrm{~b}$ \\
$\mathrm{CV}(\%)$ & & $1,09 \mathrm{~b}$ & $0,21 \mathrm{a}$ \\
\end{tabular}

Médias seguidas da mesma letra na coluna, dentro de cada profundidade, não diferem entre si pelo teste de Scott-Knott a $5 \%$. (1) Média de 15 repetições. ${ }^{(2)}$ Média de três repetições.

renovação desse sistema radicular e à atividade de microrganismos no solo associados ao processo, além da ciclagem de nutrientes.

Avaliando a Ds nos meses de agosto de 2009 e fevereiro de 2010, observaram-se valores médios de 1,06 e $1,07 \mathrm{Mg} \mathrm{m}^{-3}$, na profundidade de $0-0,05 \mathrm{~m}$, e de 1,11 e $1,13 \mathrm{Mg} \mathrm{m}^{-3}$, na profundidade de $0,20-0,25 \mathrm{~m}$, respectivamente. Avaliando a macroporosidade do solo, nos mesmos meses, observaram-se valores médios de 0,17 e $0,16 \mathrm{~m}^{3} \mathrm{~m}^{-3}$, na profundidade de 0-0,05 m, e de 0,15 e $0,14 \mathrm{~m}^{3} \mathrm{~m}^{-3}$, na profundidade de $0,20-0,25$ m, respectivamente. Esses valores não se diferenciaram estatisticamente dos encontrados no mês de maio de 2009, nessas duas profundidades, indicando que a condição estrutural manteve-se estabilizada.

Verificou-se que a variação temporal do IHO nos meses de novembro de 2008, março de 2009 e maio de 2009 foi dependente da variação da Ds (Figura 1). Com aumento na Ds do solo estudado, os valores do $\theta_{\mathrm{CC}}, \theta_{\mathrm{PMP}}$ e $\theta_{\mathrm{RP}}$ aumentaram e os valores do $\theta_{\mathrm{PA}}$ reduziram, resultados semelhantes aos encontrados por Silva et al. (1994), Leão et al. (2006) e Tormena et al. (2007), estudando solos de diferentes mineralogias e texturas.

O $\theta_{\mathrm{RP}}$ apresentou-se como limite inferior do IHO (Figura 1) nas profundidades estudadas do solo onde foram observadas restrições ao crescimento das raízes, a exemplo do que foi encontrado por Tormena et al. (1998). Para o solo em estudo, isso ocorre para valores de Ds acima de $1,18 \mathrm{Mg} \mathrm{m}^{-3}$, em que o $\theta_{\mathrm{RP}}$ manteve-se acima do $\theta_{\mathrm{PMP}}$ (Figura 1). Assim, o $\theta_{\mathrm{RP}}$ pode ser um indicativo do grau de compactação ou adensamento daquela profundidade do solo, uma vez que ocorre aumento da RP com o aumento da Ds, aliado ao aumento da coesão causado pela ação da umidade do solo entre as partículas que ficam mais próximas, à medida que ele é compactado ou adensado (Tormena et al., 2007).

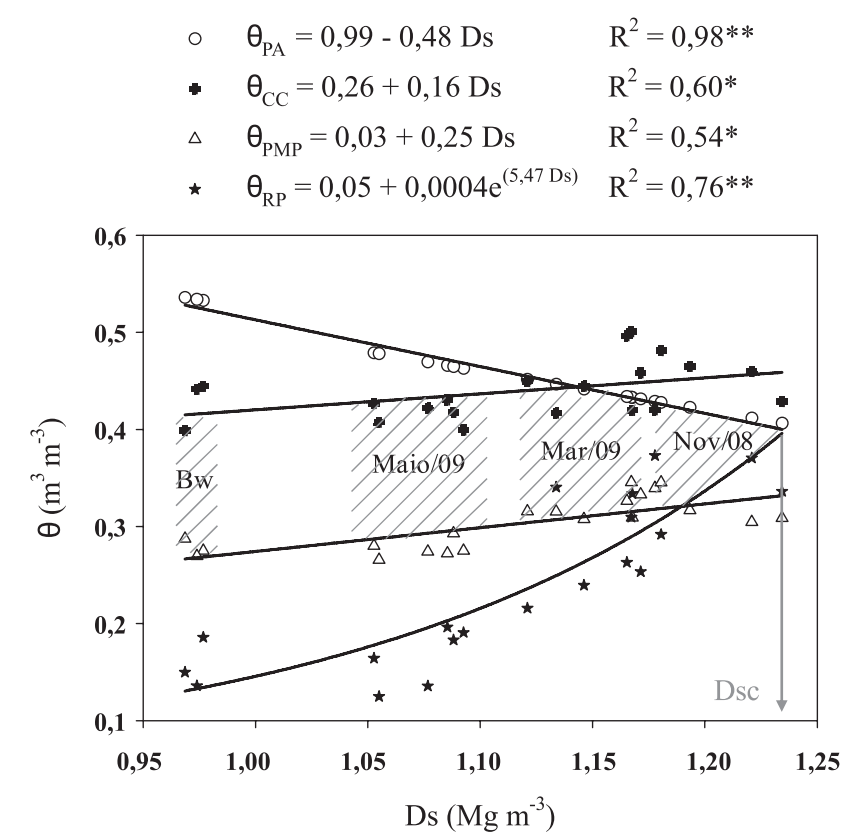

Figura 1. Variação do intervalo hídrico ótimo (área hachurada) do Latossolo Vermelho-Amarelo cultivado com Coastcross para fenação, nas profundidades de $0-0,05$ e $0,20-0,25 \mathrm{~m}$, para os meses de novembro de 2008, março de 2009 e maio de 2009, e horizonte $B w$, em função de mudanças do conteúdo de água na capacidade de campo $\left(\theta_{\mathrm{CC}}\right)$, do ponto de murcha permanente $\left(\theta_{\mathrm{PMP}}\right)$, da porosidade de aeração de $0,10 \mathrm{~m}^{3} \mathrm{~m}^{-3}$ $\left(\theta_{\mathrm{PA}}\right)$, da resistência do solo à penetração de 2,5 MPa $\left(\theta_{\mathrm{RP}}\right)$ e de alterações na densidade do solo. Dsc: densidade do solo crítica. 
Na avaliação do limite superior do IHO, observouse aumento da Ds com decréscimo do $\theta_{\mathrm{PA}}$ (Figura 1 ). $\mathrm{O}$ valor do $\theta_{\mathrm{PA}}$ manteve-se acima do $\theta_{\mathrm{CC}}$ para valores de Ds de até $1,14 \mathrm{Mg} \mathrm{m}^{-3}$; até esse limite a microestrutura está estável e preservada e verificase espaço poroso suficiente para as trocas gasosas no solo (Tormena et al., 1999). Para valores de Ds $>1,14 \mathrm{Mg} \mathrm{m}^{-3}$, observou-se redução do volume de macroporos, salientando-se que estes são efetivos na drenagem do solo. Esses resultados são semelhantes aos obtidos por outros pesquisadores na determinação do IHO (Silva et al., 1994; Imhoff et al., 2001; Leão et al., 2006; Tormena et al., 2007).

Pesquisas realizadas por Tormena et al. (1999) e Klein \& Libardi (2002) indicaram valores de Dsc próximos de $1,28 \mathrm{Mg} \mathrm{m}^{-3}$ para Latossolos de mesma textura. Na avaliação do LVA sob Coastcross em estudo, observou-se que esse limite crítico ao adequado crescimento e desenvolvimento das plantas é mais restritivo, sendo apontado valor próximo de $1,24 \mathrm{Mg} \mathrm{m}^{-3}$.

Verificou-se que 33 e $7 \%$ das amostras de solo coletadas nas profundidades de $0-0,05$ e 0,20 $0,25 \mathrm{~m}$, respectivamente, no mês de novembro de 2008, apresentaram valores de Ds superiores aos da Dsc. Para o mês de março de 2009, verificou-se que $7 \%$ das amostras coletadas na profundidade de 0,20-0,25 m mostraram valores de Ds superiores aos da Dsc. Essa restrição ao desenvolvimento de raízes nas camadas mais superficiais do solo já havia sido observada por ocasião da descrição morfológica do perfil realizada no mês de novembro de 2008, quando foi registrado um volume bem menor de raízes finas nas profundidades de $0-0,05$ e $0,20-0,25 \mathrm{~m}$, em comparação ao horizonte $\mathrm{Bw}(0,80-0,85 \mathrm{~m})$.

As variações no IHO nas profundidades de $0-0,05,0,20-0,25$ e $0,80-0,85 \mathrm{~m}(\mathrm{Bw})$ nos meses de novembro de 2008, março de 2009 e maio de 2009 são mostradas na figura 2. Avaliando os limites do IHO, observou-se que o $\theta_{\mathrm{CC}}$ representou o limite superior em maio de 2009 no horizonte $\mathrm{Bw}$, sendo o $\theta_{\mathrm{PA}}$ igual ou superior ao $\theta_{\mathrm{CC}}$ para os demais meses e profundidades. Dessa forma, a aeração foi limitante ao adequado crescimento das plantas para os meses de novembro de 2008 e março de 2009 na profundidade de 0-0,05 m, uma vez que a macroporosidade foi inferior a $0,10 \mathrm{~m}^{3} \mathrm{~m}^{-3}$ (Quadro 1). Tormena et al. (1998), ao avaliarem o IHO em Latossolo Roxo, encontraram o $\theta_{\mathrm{CC}}$ como limite superior do IHO em $97 \%$ das amostras avaliadas, contrapondo aos resultados apresentados neste trabalho e confirmando o elevado grau de degradação da estrutura do solo aqui verificado para os meses de novembro de 2008 e março de 2009.

No mês de novembro de 2008, verificou-se redução da qualidade estrutural do LVA estudado, quantificada pelas substituições do $\theta_{\mathrm{PMP}}$ pelo $\theta_{\mathrm{RP}}$ e do $\theta_{\mathrm{CC}}$ pelo $\theta_{\mathrm{PA}}$ como limite inferior e superior do IHO na profundidade de $0-0,05 \mathrm{~m}$ e do $\theta_{\text {PMP }}$ pelo $\theta_{\mathrm{RP}}$ como limite inferior do IHO na profundidade de 0,20-0,25 m. Essa redução da qualidade estrutural tem reflexos negativos na quantidade de água potencialmente disponível para as plantas (Figura 2).

A melhoria da estrutura do solo, como era de se esperar, ocorreu de forma gradativa, sendo verificado melhor valor do $\theta_{\mathrm{RP}}$ e do $\theta_{\mathrm{PA}}$ quando se comparam os meses de novembro 2008 e março de 2009 e os meses de março e maio de 2009. A evolução estrutural é constatada pelo aumento do IHO de 0,048, 0,092 e $0,149 \mathrm{~m}^{3} \mathrm{~m}^{-3}$ na profundidade de $0-0,05 \mathrm{~m}$ e de $0,070,0,123$ e $0,132 \mathrm{~m}^{3} \mathrm{~m}^{-3}$ na profundidade de 0,20 $0,25 \mathrm{~m}$, para os meses de novembro de 2008 e março e maio de 2009, respectivamente, em comparação ao valor de $0,151 \mathrm{~m}^{3} \mathrm{~m}^{-3}$ obtido no horizonte $\mathrm{Bw}$ (Figura 2).
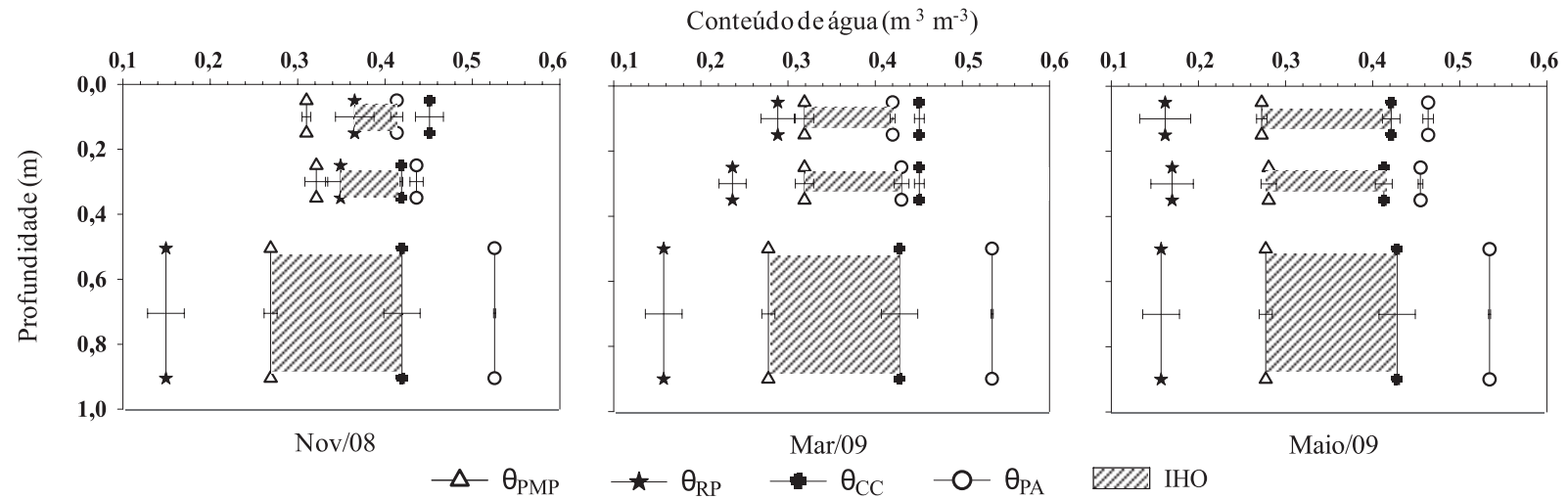

Figura 2. Limites inferiores e superiores do intervalo hídrico ótimo (IHO), nos limites críticos de capacidade de campo $\left(\theta_{\mathrm{CC}}\right)$, ponto de murcha permanente $\left(\theta_{\mathrm{PMP}}\right)$, porosidade de aeração de $\mathbf{0 , 1 0} \mathbf{~ m}^{3} \mathrm{~m}^{-3}\left(\theta_{\mathrm{PA}}\right)$ e resistência do solo à penetração de $2,5 \mathrm{MPa}\left(\theta_{\mathrm{RP}}\right)$, para o Latossolo Vermelho-Amarelo cultivado com Coastcross para fenação, nas profundidades de $0-0,05,0,20-0,25$ e $0,80-0,85 \mathrm{~m}(\mathrm{Bw})$, nos meses de novembro de 2008, março de 2009 e maio de 2009. 
A melhoria na disponibilidade hídrica do solo foi maior na profundidade de $0-0,05 \mathrm{~m}$ em comparação com a de 0,20-0,25 m, fato atribuído à maior atividade biológica (sistema radicular e atividade dos microrganismos) nessas profundidades, que provavelmente potencializaram a formação de bioporos com ampla variação de tamanho (Willians \& Weil, 2004).

As alterações estruturais entre os meses de novembro de 2008 e maio de 2009 são indicadas pelo aumento médio de $80 \%$ na macroporosidade, pela redução média de $10 \%$ nos valores de Ds, pela redução média de $65 \%$ na RP e pelo aumento médio de $140 \%$ na disponibilidade hídrica, quando se consideram as duas primeiras profundidades do LVA cultivado com Coastcross para fenação.

A melhoria da estruturação do solo, influenciada pelo crescimento e renovação do sistema radicular associados à atividade de microrganismos, melhorou os atributos físico-hídricos do LVA, ou seja, aumentou a capacidade de armazenamento de água, reduziu a resistência do solo à penetração de raízes e favoreceu a aeração. Isso, sem dúvida, contribui para o restabelecimento do seu potencial produtivo.

\section{CONCLUSÕES}

1. O intervalo hídrico ótimo revelou-se um adequado indicador da alteração da estrutura do Latossolo.

2. O Coastcross apresenta potencial para a melhoria da estrutura de Latossolo degradado fisicamente, sendo sugerida sua utilização como alternativa ao uso de implementos agrícolas nesse contexto.

3. A Dsc de $1,24 \mathrm{Mg} \mathrm{m}^{-3}$ é limitante ao adequado crescimento e desenvolvimento da forrageira no solo em estudo.

\section{AGRADECIMENTO}

Agradecemos à Fundação de Amparo à Pesquisa do Estado de Minas Gerais - FAPEMIG, pelo apoio financeiro na realização desta pesquisa.

\section{LITERATURA CITADA}

BENGOUGH, A.G.; BRANSBY, M.F.; HANS, J.; McKENNA, S.J.; ROBERTS, T. \& VALENTINE, T.A. Root responses to soil physical conditions: Growth dynamics from field to cell. J. Exper. Bot., 57:437-443, 2006.
CAMARGO, O.A. \& ALLEONI, L.R.F. Compactação do solo e o desenvolvimento de plantas. Piracicaba, Escola Superior de Agricultura Luiz de Queiroz, 1997. 132p.

CHAGAS, C.S.; CURI, N.; DUARTE, M.N.; MOTTA, P.E.F. \& LIMA, J.M. Orientação das camadas de rochas metapelíticas pobres na gênese de Latossolos sob Cerrado. Pesq. Agropec. Bras., 32:539-548, 1997.

EMPRESA BRASILEIRA DE PESQUISA AGROPECUÁRIA - EMBRAPA. Centro Nacional de Pesquisas de Solos. Manual de métodos de análises de solo. 2.ed. Rio de Janeiro, 1997. 212p.

EMPRESA BRASILEIRA DE PESQUISA AGROPECUÁRIA - EMBRAPA. Centro Nacional de Pesquisas de Solos. Sistema brasileiro de classificação de solos. 2.ed. Rio de Janeiro, 2006. 306p.

FERREIRA, D.F. Estatística básica. Lavras, Universidade Federal de Lavras, 2005. 654p.

GIAROLA, N.F.B.; TORMENA, C.A. \& DUTRA, A.C. Degradação física de um Latossolo Vermelho utilizado para produção intensiva de forragem. R. Bras. Ci. Solo, 31:863-873, 2007.

IMHOFF, S.; SILVA, A.P.; DIAS JÚNIOR, M.S. \& TORMENA, C.A. Quantificação de pressões críticas para o crescimento das plantas. R. Bras. Ci. Solo, 25:11-18, 2001.

JIMENEZ, R.L.; GONÇALVES, W.G.; ARAÚJO FILHO, J.V.; ASSIS, R.L.; PIRES, F.R. \& SILVA, G.P. Crescimento de plantas de cobertura sob diferentes níveis de compactação em um Latossolo Vermelho. R. Bras. Eng. Agric. Amb., 12:116-121, 2008.

KAISER, D.R.; REINERT, D.J.; REICHERT, J.M. \& KUNZ, M. Intervalo hídrico ótimo no perfil explorado pelas raízes de feijoeiro em um Latossolo sob diferentes níveis de compactação. R. Bras. Ci. Solo, 33:845-855, 2009.

KLEIN, V.A. \& LIBARDI, P.L. Densidade e distribuição do diâmetro dos poros de um Latossolo Vermelho, sob diferentes sistemas de uso e manejo. R. Bras. Ci. Solo, 26:857-867, 2002.

LEÃO, T.P.; SILVA, A.P.; MACEDO, M.C.M.; IMHOFF, S. \& EUCLIDES, V.P.B. Least limiting water range: A potential indicator of changes in near-surface soil physical quality after the conversion of Brazilian Savanna into pasture. Soil Tillage Res., 88:279-285, 2006.

LEÃO, T.P.; SILVA, A.P.; PERFECT, E. \& TORMENA, C.A. An algorithm for calculating the least limiting water range of soil using SAS. Agron. J., 97:1210-1215, 2005.

LETEY, J. Relationship between soil physical properties and crop productions. Adv. Soil Sci., 1:277-294, 1985.

LIMA, V.M.P.; OLIVEIRA, G.C.; SEVERIANO, E.C. \& OLIVEIRA, L.F.C. Intervalo hídrico ótimo e porosidade de solos cultivados em área de proteção ambiental do Sul de Minas Gerais. R. Bras. Ci. Solo, 33:1087-1095, 2009.

MAGALHÃES, E.N.; OLIVEIRA, G.C.; SEVERIANO, E.C.; COSTA, K.A.P. \& CASTRO, M.B. Recuperação estrutural e produção de capim-tifton 85 em um Argissolo VermelhoAmarelo compactado. Ci. Anim. Bras., 10:68-76, 2009. 
MEDEIROS, J.C.; SILVA, A.P.; CERRI, C.E.P.; GIAROLA, N.F.B.; FIGUEIREDO, G.C. \& FRACETTO, F.J.C. Linking physical quality and $\mathrm{CO}_{2}$ emissions under long-term no-till and conventional-till in a subtropical soil in Brazil. Plant Soil, 338:5-15, 2011.

MULLER, M.M.L.; CECCON, G. \& ROSOLEM, C.A. Influência da compactação do solo em subsuperfície sobre o crescimento aéreo e radicular de plantas de adubação verde de inverno. R. Bras. Ci. Solo, 25:531-538, 2001.

OLIVEIRA, G.C.; DIAS JUNIOR, M.S.; CURI, N. \& RESCK, D.V.S. Caracterização química e físico-hídrica de um Latossolo Vermelho após 20 anos de manejo e cultivo do solo. R. Bras. Ci. Solo, 28:335-344, 2004.

REICHERT, J.M.; SUZUKI, L.E.A.S.; REINERT, D.J.; HORN, R. \& HAKANSSON, I. Reference bulk density and critical degree-of-compactness for no-till crop production in subtropical highly weathered soils. Soil Tillage Res., 102:242-254, 2009.

RICHARD, G.; COUSIN, I.; SILLON, J.F.; BRUAND, A. \& GUÉRIF, J. Effects of compaction on the porosity of a silt soil: Influence on unsaturated hydraulic properties. Eur. J. Soil Sci., 52:49-58, 2001.

SANTOS, R.D.; LEMOS, R.C.; SANTOS, H.G.; KER, J.C. \& ANJOS, L.H.C. Manual de descrição e coleta de solo no campo. 5.ed. Viçosa, MG, Sociedade Brasileira de Ciência do Solo, 2005. 92p.

SEVERIANO, E.C.; OLIVEIRA, G.C.; DIAS JUNIOR, M.S.; COSTA, K.A.P.; CASTRO, M.B. \& MAGALHÃES, E.N. Potencial de descompactação de um Argissolo promovido pelo capim-tifton 85. R. Bras. Eng. Agric. Amb., 14:39-45, 2010.
SIGMA PLOT. Scientific Graphing Software. Versão 10.0. San Rafael, Jandel Corporation, 2006.

SILVA, A.P.; KAY, B.D. \& PERFECT, E. Characterization of the least limiting water range. Soil Sci. Soc. Am. J., 58:17751781, 1994.

SILVA, I.F. \& MIELNICZUK, J. Sistemas de cultivo e características do solo afetando a estabilidade de agregados. R. Bras. Ci. Solo, 22:311-317, 1997.

TORMENA, C.A.; ARAÚJO, M.A.; FIDALSKI, J. \& COSTA, J.M. Variação temporal do intervalo hídrico ótimo de um Latossolo Vermelho distroférrico sob sistemas de plantio direto. R. Bras. Ci. Solo, 31:211-219, 2007.

TORMENA, C.A.; SILVA, A.P. \& LIBARDI, P.L. Caracterização do intervalo hídrico ótimo de um Latossolo Roxo sob plantio direto. R. Bras. Ci. Solo, 22:573-581, 1998.

TORMENA, C.A.; SILVA, A.P. \& LIBARDI, P.L. Soil physical quality of a Brazilian Oxisol under two tillage systems using the least limiting water range approach. Soil Tillage Res., 52:223-232, 1999 .

van GENUCHTEN, M.T. A closed-form equation for predicting the hydraulic conductivity of unsaturated soils. Soil Sci. Soc. Am. J., 44:892-898, 1980.

WILLIAMS, S.M. \& WEIL, R.R. Crop cover root channels may alleviate soil compaction effects on soybean crop. Soil Sci. Soc. Am. J., 68:1403-1409, 2004.

WU, L.; FENG, G.; LETEY, J.; FERGUSON, L.; MITCHELL, J.; McCULLOUGH-SANDEN, B. \& MARKEGARD, G. Soil management effects on the nonlimiting water range. Geoderma, 114:401-414, 2003. 